\title{
Qualidade de vida de quem cuida de portadores de demência com corpos de Lewy
}

\author{
Quality of life of caregivers of patients with Lewy body dementia
}

Eberson Rodrigues do Nascimento', Maria Alves Barbosa², Virginia Visconde Brasili , Ana Luiza Lima Sousa4, Geraldo Francisco do Amaral', Paula Jardim Jácomo ${ }^{6}$

\section{RESUMO}

Introdução: Cuidar de portadores de demência pode provocar impacto na qualidade de vida dos familiares. Objetivo: Verificar a qualidade de vida de familiares que cuidam de portadores de demência com corpos de Lewy (DCL). Método: Estudo transversal, realizado com 90 familiares de portadores de DCL atendidos em hospital universitário de Goiânia/GO (Brasil), que responderam ao instrumento de avaliação de qualidade de vida WHOQOL-BREF. Resultados: Os entrevistados eram 53,3\% homens, na maioria casados e com ensino médio de escolaridade, com idade média de 47,4 \pm 13,8 anos e média de tempo como cuidador de 13,9 \pm 9,3 meses. O domínio Meio Ambiente obteve maior média $(26,92 \pm 5,88)$ e o Psicológico $(19,66 \pm 4,32)$ e Relação social $(9,84 \pm 2,18)$, as menores. A média dos domínios foi influenciada pelo sexo e pelo tempo que o familiar era cuidador. Conclusão: Atenção especial deve ser dada às alterações na saúde do cuidador, para que ele não se torne um "paciente oculto" e incapaz de lidar com as demandas do portador de DCL. O conhecimento das especificidades e compreensão dos sintomas da patologia auxiliam na adequação dos recursos pessoais para enfrentar as alterações comportamentais, apontadas como o fator mais impactante na vida do cuidador.

\section{ABSTRACT}

Introduction: Taking Taking care of people with dementia may have an impact on the quality of life for family members. Objective: To verify the quality of life for family members who take care of people with dementia with Lewy bodies (DLB). Method: A cross-sectional study with 90 relatives of patients with DLB treated in a University hospital in Goiânia/GO (Brazil), who responded to the instrument of evaluation for the quality of life WHOQOL-BREF. Results: From the people who were interviewed, 53.3\% were male, most married and high school graduated, average age of $47.4 \pm 13.8$ years and average time as a care taker $13.9 \pm 9.3$ months. The Environment Domain got the highest average (26.92 \pm 5.88$)$, the Psychological $(19,66 \pm 4,32)$ and the Social Relationship $(9.84 \pm 2.18)$ the lowest. The average for the domains was influenced by genus and by the time that the family member was a care taker. Conclusion: Special attention should be given to changes in the health of caretakers, so they

1 Universidade Federal de Goiás (UFG), Programa de Pós-Graduação em Ciências da Saúde. 2 Universidade Federal do Rio de Janeiro (UFRJ); Universidade de São Paulo (USP); UFG. 3 USP; UFG. 6 UFG. 


\section{Keywords}

Quality of life, Lewy body

disease, caretakers. do not become a "hidden patient", and unable to deal with the demands of patients with DLB. The knowledge and understanding of the specific symptoms of the pathology helps to accommodate the human resources to face the behavioral changes, identified as the most impacting factor in the lives of caretakers.

\section{INTRODUÇÃO}

O declínio cognitivo que ocorre com o aumento da idade é uma das grandes preocupações dos profissionais de saúde que trabalham com o envelhecimento humano. Dados estatísticos revelam que a quantidade de casos de demência aumenta com o avanço da idade em todo o mundo, mostrando uma prevalência média, acima de 65 anos de idade, variando de 2,2\% na África, 5,7\% na Ásia, 6,2\% na América do Norte, 7,1\% na América do Sul, até 9,0\% na Europa'.

O número de pessoas acima de 60 anos ou mais com demência nos países em desenvolvimento no ano 2000 era de aproximadamente 11 milhões, e no Brasil, de 390 mil pessoas².

A demência representa um grupo heterogêneo de doenças que têm em comum a alteração das funções cognitivas - memória, linguagem, práxis, capacidade de reconhecer e identificar objetos, abstração, organização, capacidade de planejamento e sequenciamento de atos ${ }^{3}$. É causada por múltiplas etiologias, tem caráter permanente e progressivo ou transitório, acarretando repercussões sociais e ocupacionais ao portador ${ }^{4}$.

Lewy $(1912)^{5}$ identificou, entre um grupo de portadores de doença de Parkinson, alguns sintomas inespecíficos como perda da memória, sintomas psiquiátricos e flutuação no desempenho cognitivo e no nível de consciência, hipotetizando que esses sintomas poderiam compor um quadro demencial diferente, denominado demência com corpos de Lewy. Com o passar do tempo, foram confirmados, além do comprometimento cognitivo, sintomas neurológicos (parkinsonismo, distúrbios do sono, falência autonômica), psiquiátricos (alucinações, alterações comportamentais, sintomas depressivos) e clínicos em geral (síncope, hipotensão postural, quedas) $)^{6,7}$.

O agravamento das alterações de humor e do comportamento acaba por interferir na vida cotidiana do portador e determina a necessidade de acompanhamento de um cuidador ${ }^{8}$. Muitos desses cuidadores têm parentesco com o portador de demência, podendo haver conflito e desagregação na estrutura familiar pela sobrecarga, e refletir na qualidade de vida dos familiares ${ }^{9}$.

A qualidade de vida de pessoas que cuidam de portadores de demência com corpos de Lewy e convivem com eles ainda se mostra um objeto de estudo pouco explorado, justificando a realização de estudos para subsidiar os profissionais sobre os aspectos que demandam orientação profissional com a intenção de amenizar o impacto de cuidar de um portador de demência.
Como qualidade de vida é um constructo subjetivo e multidimensional e foi definida pela Organização Mundial da Saúde (OMS) como "a percepção do indivíduo sobre sua posição na vida, no contexto da cultura e do sistema de valores nos quais ele vive e em relação aos seus objetivos, expectativas, padrões e preocupações"10, sua avaliação pressupõe perguntar ao sujeito como ele avalia o que está acontecendo na sua vida.

O estudo teve como objetivo geral avaliar a qualidade de vida de familiares que convivem com portadores de demência com corpos de Lewy e cuidam deles. Foram objetivos específicos: identificar o perfil sociodemográfico de familiares de portadores de demência com corpos de Lewy; analisar o valor atribuído pelos familiares de portadores de demência com corpos de Lewy aos domínios do instrumento de avaliação de qualidade de vida WHOQOL-BREF; e comparar os estratos das variáveis sociodemográficas à média dos escores dos domínios do WHOQOL-BREF, atribuídos pelos familiares de portadores de demência com corpos de Lewy.

\section{MÉTODO}

Estudo transversal, realizado no ambulatório do Hospital das Clínicas da Universidade Federal de Goiás (HC-UFG).

A população constituiu-se dos familiares de portadores de demência com corpos de Lewy, cadastrados e em tratamento no Serviço de Neurologia Cognitiva Comportamental do Núcleo de Neurociências do HC-UFG. O diagnóstico de $\mathrm{DCL}$ foi realizado por dois neurologistas e um psiquiatra segundo os critérios de McKeith et al." (Tabela 1), durante o ano de 2010. Todos os pacientes apresentavam, além das características essenciais para o diagnóstico, dois critérios centrais e pelo menos um dos sugestivos.

Foram considerados familiares cuidadores os indivíduos que conviviam diariamente com portador de demência com corpos de Lewy e prestavam cuidados a ele. Esses cuidadores foram identificados no ambulatório quando acompanhavam o portador de demência em consulta de rotina agendada no período da coleta de dados, sendo convidados a participarem do estudo. Sua anuência foi registrada com a assinatura do Termo de Consentimento Livre e Esclarecido. Aqueles que concordaram em participar foram visitados pelo pesquisador em seus domicílios, quando foi realizada a coleta de dados. Para essa visita foi feito aprazamento de acordo com o horário mais conveniente para a família. 
Tabela 1. Critérios diagnósticos de demência com corpos de Lewy

1. Características essenciais para o diagnóstico de possivel ou provável DCL:
Declínio cognitivo progressivo com magnitude suficiente para interferir no
funcionamento social e ocupacional do paciente
Comprometimento grave ou persistente da memória não ocorre necessariamente nas
fases iniciais da DCL, mas se torna evidente com a evolução na maioria dos casos
Déficits de atenção, de habilidades frontosubcorticais e visuoespaciais podem ser
especialmente proeminentes

2. Duas das seguintes características centrais são necessárias para o diagnóstico de DCL provável e uma para o diagnóstico de DCL possivel:

Cognição flutuante com oscilações marcantes da atenção e do estado de alerta

Alucinaçōes visuais recorrentes que são tipicamente bem formadas e detalhadas

Sinais de parkinsonismo espontâneo

\section{Características sugestivas de $D C L$ :}

Transtornos do comportamento associados ao sono REM

Sensibilidade aos neurolépticos

SPECT ou PET com captação reduzida de dopamina nos núcleos da base

\section{Caracteristicas de suporte (que corroboram o diagnóstico) de DCL:}

Quedas repetidas e síncope

Perdas transitórias da consciência

Disfunção autonômica

Ilusões sistematizadas

Depressão

5. Características (de exclusão) que tornam menos provável o diagnóstico de DCL:

História de acidentes vasculares cerebrais

Outras doenças sistêmicas ou neurológicas que interferem na performance cognitiva

A amostra foi composta por 90 familiares cuidadores maiores de 18 anos. Foram excluídos aqueles cuidadores com idade inferior a 18 anos e/ou cujo familiar não preenchia os critérios para demência com corpos de Lewy.

Foram utilizados: um questionário sociodemográfico contendo variáveis referentes a sexo, idade, tempo de cuidado ao paciente, escolaridade e estado civil, e o Questionário de Avaliação da Qualidade de Vida - WHOQOL-BREF, proposto pela OMS ${ }^{10}$.

É um instrumento validado no Brasil que contém 26 questões, duas questões gerais sobre qualidade de vida e as 24 demais abordando quatro domínios da qualidade de vida, divididos em 24 facetas (Tabela 2) ${ }^{12}$.

Os dados foram digitados em planilha eletrônica do programa Microsoft Office Excel e analisados com a utilização do SPSS [Statistical Package for the Social Sciences (SPSS), versão 17.0].

As variáveis quantitativas foram apresentadas em valores absolutos e médias, com sua variabilidade identificada pelo desvio-padrão e valores máximos e mínimos. As variáveis categóricas foram apresentadas segundo as proporções. Para a análise de associação entre as variáveis categóricas, utilizouse o teste qui-quadrado. Foi utilizado para análise do padrão de distribuição das variáveis quantitativas o teste de Kolmogorov-Smirnov.

Para verificação da existência de diferença entre as médias nos domínios em relação às variáveis sociodemográficas e tempo que o familiar era cuidador, foram utilizados os
Tabela 2. Domínios e facetas do WHOQOL-BREF

\begin{tabular}{|c|c|}
\hline Domínios & Facetas \\
\hline Físico & $\begin{array}{l}\text { 1. Dor e desconforto } \\
\text { 2. Energia e fadiga } \\
\text { 3. Sono e repouso } \\
\text { 9. Mobilidade } \\
\text { 10. Atividades da vida cotidiana } \\
\text { 11. Dependência de medicação ou de tratamentos } \\
\text { 12. Capacidade de trabalho }\end{array}$ \\
\hline Psicológico & $\begin{array}{l}\text { 4. Sentimentos positivos } \\
\text { 5. Pensar, aprender, memória e concentração } \\
\text { 6. Autoestima } \\
\text { 7. Imagem corporal e aparência } \\
\text { 8. Sentimentos negativos } \\
\text { 24. Espiritualidade/religião/crenças pessoais }\end{array}$ \\
\hline Relações sociais & $\begin{array}{l}\text { 13. Relações pessoais } \\
\text { 14. Suporte (Apoio) social } \\
\text { 15. Atividade sexual }\end{array}$ \\
\hline Meio ambiente & $\begin{array}{l}\text { 16. Segurança física e proteção } \\
\text { 17. Ambiente no lar } \\
\text { 18. Recursos financeiros } \\
\text { 19. Cuidados de saúde e sociais: disponibilidade e qualidade } \\
\text { 20. Oportunidades de adquirir novas informações e habilidades } \\
\text { 21. Participação em e oportunidades de recreação/lazer } \\
\text { 22. Ambiente físico: (poluição/ruído/trânsito/clima) } \\
\text { 23. Transporte }\end{array}$ \\
\hline
\end{tabular}

testes de Mann-Whitney-U e Kruskal-Wallis. Os testes de correlação foram o índice de Pearson ou Spearman. Para todos os testes o nível de significância estatística foi estabelecido em $5 \%$.

Para o cálculo dos escores da qualidade de vida do WHOQOL-BREF, foram seguidas as recomendações de sintaxe padronizada ${ }^{10}$, usando escores com valores de 4 a 20, transformados em uma escala tipo Likert variando de 0 a 100. As respostas foram recodificadas, de maneira que todas tivessem o mesmo significado numérico (quanto menor o valor, pior a situação). As questões Q3, Q4 e Q26 são originalmente formuladas com valores na direção oposta (quanto maior o escore mais desfavorável a situação), e assim foi feita inversão para composição final do escore $(1=5 ; 2=4 ; 3=3$; $4=2 ; 5=1)$.

Não se calculou escore total de QV por ser um constructo multidimensional, tendo cada domínio um escore independente, apresentado por meio da média e do desvio-padrão. Foi avaliada a consistência interna do WHOQOL-BREF pelo coeficiente de fidedignidade de Cronbach para cada domínio.

O projeto de pesquisa foi submetido à avaliação do Comitê de Ética em Pesquisa do Hospital das Clínicas da Universidade Federal de Goiás, obtendo-se parecer favorável sob Protocolo no 098/2010. Todos os participantes assinaram o Termo de Consentimento Livre e Esclarecido antes de qualquer procedimento do estudo. 


\section{RESULTADOS}

Em média, os cuidadores levaram 10 minutos para o preenchimento do questionário. Eles eram na maioria homens, casados, com idade entre 18 e 92 anos, sendo a idade média 47,4 $\pm 13,4$ anos. Quanto à escolaridade, 38,9\% eram analfabetos e cuidadores, em média, há 13,9 ( \pm 7,93) meses (Tabela 3).

As médias dos domínios da qualidade de vida do WHOQOL-BREF variaram entre 26,92 \pm 5,88 do domínio Meio Ambiente e 9,84 $\pm 2,18$ do domínio Relação Social. A confiabilidade do instrumento foi avaliada por meio do alfa de Cronbach, que variou entre 0,670 e 0,926 (Tabela 4).

As respostas às questões 2 e 3 do WHOQOL-BREF (overal) sobre avaliação da qualidade de vida e satisfação com a própria saúde obtiveram média de 12,8 $( \pm 2,1)$.

Nas respostas às demais questões do WHOQOL-BREF, foi evidenciado que, no domínio Físico, contribuíram para elevar a média desse domínio os itens: "O quanto você precisa de algum tratamento médico para levar sua vida diária?", "Em que medida você acha que sua dor (física) impede você de fazer o que você precisa?" e "Você tem energia suficiente para seu dia a dia?" (Tabela 5).

Tabela 3. Caracterização sociodemográfica de 90 familiares que cuidam de portadores de demência com corpos de Lewy Goiânia/GO, 2010

\begin{tabular}{|c|c|c|c|}
\hline Características sociodemográficas & N & $\%$ & $p^{*}$ \\
\hline Sexo & & & 0,527 \\
\hline Masculino & 48 & 53,3 & \\
\hline Feminino & 42 & 46,7 & \\
\hline Idade média (faixa etária) & \multicolumn{2}{|c|}{47,4 ( $\pm 13,4$ anos) } & 0,000 \\
\hline$\leq 30$ anos & 10 & 11,1 & \\
\hline $30---60$ anos & 65 & 72,2 & \\
\hline$>60$ anos & 15 & 16,7 & \\
\hline Tempo como cuidador (meses) & \multicolumn{2}{|c|}{ Média 13,9 ( $\pm 7,93)$} & 0,000 \\
\hline$\leq 6$ meses & 11 & 12,2 & \\
\hline $6---\mid 12$ meses & 26 & 28,9 & \\
\hline $12---\mid 18$ meses & 34 & 37,8 & \\
\hline $18---\mid 24$ meses & 15 & 16,7 & \\
\hline$>24$ meses & 4 & 4,4 & \\
\hline Escolaridade & & & 0,000 \\
\hline Analfabeto & 35 & 38,9 & \\
\hline Ensino fundamental & 16 & 17,8 & \\
\hline Ensino médio & 36 & 40,0 & \\
\hline Ensino superior & 3 & 3,3 & \\
\hline Estado civil & & & 0,000 \\
\hline Solteiro & 10 & 11,1 & \\
\hline Casado & 73 & 81,1 & \\
\hline
\end{tabular}

Tabela 4. Médias, desvios-padrão e coeficiente de fidedignidade dos domínios do WHOQOL-BREF, aplicados a 90 cuidadores de portadores de demência com corpos de Lewy - Goiânia/GO, 2010

\begin{tabular}{lcc}
\hline Domínios WHOQOL-BREF* & Média \pm DP & $\alpha$-Cronbach \\
\hline I - Físico & $23,10 \pm 3,70$ & 0,670 \\
II - Psicológico & $19,66 \pm 4,32$ & 0,844 \\
III - Relação Social & $9,84 \pm 2,18$ & 0,818 \\
IV - Meio Ambiente & $26,92 \pm 5,88$ & 0,896 \\
OVERALL & $12,8 \pm 2,1$ & 0,926 \\
${ }^{*} \mathrm{P}=0,710$. &
\end{tabular}

Tabela 5. Média das respostas às questões dos domínios do WHOQOL-BREF referidas por 90 cuidadores de portadores de demência com corpos de Lewy - Goiânia/GO, 2010

\begin{tabular}{|c|c|}
\hline Questões/Domínios WHOQOL & Média $\pm D P$ \\
\hline \multicolumn{2}{|l|}{ DOMÍNIO FísICO } \\
\hline $\begin{array}{l}\text { 3) Em que medida você acha que sua dor (física) impede você de fazer } 0 \\
\text { que você precisa? }\end{array}$ & $3,56 \pm 1,21$ \\
\hline $\begin{array}{l}\text { 4) } 0 \text { quanto você precisa de algum tratamento médico para levar sua vida } \\
\text { diária? }\end{array}$ & $3,65 \pm 1,25$ \\
\hline 10) Você tem energia suficiente para seu dia a dia? & $3,55 \pm 0,79$ \\
\hline 15) Quão bem você é capaz de se locomover? & $3,38 \pm 0,84$ \\
\hline 16) Quão satisfeito(a) você está com o seu sono? & $3,40 \pm 0,77$ \\
\hline $\begin{array}{l}\text { 17) Quão satisfeito(a) você está com sua capacidade de desempenhar as } \\
\text { atividades do seu dia a dia? }\end{array}$ & $3,32 \pm 0,81$ \\
\hline 18) Quão satisfeito(a) você está com sua capacidade para o trabalho? & $3,51 \pm 0,89$ \\
\hline \multicolumn{2}{|l|}{ DOMÍNIO PSICOLÓGICO } \\
\hline 5) 0 quanto você aproveita a vida? & $3,06 \pm 0,99$ \\
\hline 6) Em que medida você acha que a sua vida tem sentido? & $3,07 \pm 0,93$ \\
\hline 7) 0 quanto você consegue se concentrar? & $3,20 \pm 0,89$ \\
\hline 11) Você é capaz de aceitar sua aparência física? & $3,59 \pm 0,90$ \\
\hline 19) Quão satisfeito(a) você está consigo mesmo? & $3,50 \pm 0,91$ \\
\hline $\begin{array}{l}\text { 26) Com que frequência você tem sentimentos negativos tais como mau } \\
\text { humor, desespero, ansiedade, depressão? }\end{array}$ & $4,08 \pm 0,93$ \\
\hline \multicolumn{2}{|l|}{ DOMÍNIO AMBIENTAL } \\
\hline 8) Quão seguro(a) você se sente em sua vida diária? & $3,28 \pm 0,93$ \\
\hline 9) Quão saudável é o seu ambiente físico (clima, barulho, poluição, atrativos)? & $3,28 \pm 0,98$ \\
\hline 12) Você tem dinheiro suficiente para satisfazer suas necessidades? & $3,31 \pm 1,01$ \\
\hline 13) Quão disponíveis para você estão as informações que precisa no seu dia a dia? & $3,55 \pm 0,92$ \\
\hline 14) Em que medida você tem oportunidades de atividade de lazer? & $3,58 \pm 0,96$ \\
\hline 23) Quão satisfeito(a) você está com as condições do local onde mora? & $3,35 \pm 0,83$ \\
\hline 24) Quão satisfeito(a) você está com o seu acesso aos serviços de saúde? & $3,43 \pm 0,93$ \\
\hline 25) Quão satisfeito(a) você está com o seu meio de transporte? & $3,53 \pm 0,92$ \\
\hline
\end{tabular}

Contribuíram para elevar a média do domínio Psicológico as respostas das questões frequência de sentimentos negativos como mau humor, desespero, ansiedade, depressão e aceitar sua aparência física (Tabela 5). 
As médias dos escores dos domínios do WHOQOL-BREF foram analisadas em relação aos estratos das variáveis do estudo e não foi identificada diferença significativa entre a média de escores nos domínios Psicológico e Meio Ambiente em relação a sexo, idade, estado civil e faixa de escolaridade. No entanto, houve diferença significativa na distribuição das médias dos escores dos domínios Físico e Relações Sociais em relação à variável sexo.

Outra variável que esteve associada com os escores médios foi o tempo em que o indivíduo era cuidador. Somente os escores do domínio Físico não estiveram associados significativamente com essa variável. No domínio Psicológico, aqueles com 18 a 24 meses como cuidadores apresentaram melhores médias (22,80; $p=0,017)$, ocorrendo o mesmo nos domínios Relações Sociais $(10,93 ; p=0,030)$ e Meio Ambiente $(31,60 ; p=0,013)$ (Tabela 6).

No domínio Relações Sociais as mulheres apresentaram escore médio menor $(p=0,027)$ em relação aos homens. E no domínio Físico também as mulheres apresentaram meIhor escore médio $(p=0,017)$ (Tabela 6$)$.

$\mathrm{Na}$ análise de correlação entre os escores dos domínios que apresentaram associação significativa com a variável "Tempo como Cuidador" (domínios Psicológico, Relação Social e Meio Ambiente), foi encontrada correlação significativa nos três domínios citados, $p=0,017$ no Psicológico, 0,030 no Relação Social e 0,013 no Meio Ambiente (Figuras 1 e 2).

A correlação da idade em todos os domínios foi negativa, ou seja, na medida em que aumentava a idade, diminuía a média do escore de qualidade de vida. No entanto, não foi encontrado nível de significância na análise de correlação.

Quanto ao sexo, houve correlação significativa nos domínios Físico ( $p=0,017)$ e Relação Social $(p=0,027)$.

Não houve covariação entre o tempo que o familiar é cuidador e o sexo ou qualquer outra variável.

Tabela 6. Médias e desvios-padrão dos domínios do WHOQOL-BREF em relação aos estratos das variáveis sociodemográficas de cuidadores de portadores de demência com corpos de Lewy - Goiânia/GO, 2010 ( $n=90$ )

\begin{tabular}{|c|c|c|c|c|c|c|c|c|}
\hline \multirow{3}{*}{ Variáveis } & \multicolumn{8}{|c|}{ Domínios } \\
\hline & \multicolumn{2}{|c|}{ Físico } & \multicolumn{2}{|c|}{ Psicológico } & \multicolumn{2}{|c|}{ Relaçōes Sociais } & \multicolumn{2}{|c|}{ Meio Ambiente } \\
\hline & Média $\pm D P$ & $p$ & Média \pm DP & $\mathbf{P}$ & Média $\pm D P$ & $\mathrm{p}$ & Média \pm DP & $p$ \\
\hline Sexo & & $0,017^{*}$ & & 0,123 & & $0,027^{*}$ & & 0,246 \\
\hline Feminino & $24,90 \pm 3,92$ & & $20,38 \pm 4,47$ & & $9,42+2,21$ & & $27,50 \pm 5,85$ & \\
\hline Masculino & $23,19 \pm 3,34$ & & $19,02 \pm 4,13$ & & $10,33+2,07$ & & $26,42 \pm 5,93$ & \\
\hline Faixa etária & & 0,124 & & 0,249 & & 0,241 & & 0,129 \\
\hline$\leq 30$ & $24,00 \pm 4,78$ & & $19,90 \pm 3,04$ & & $10,40+2,12$ & & $27,70 \pm 6,58$ & \\
\hline $30-60$ & $24,40+3,65$ & & $20,05+4,66$ & & $9,98+2,14$ & & $27,55+5,80$ & \\
\hline$\geq 60$ & $22,20+2,73$ & & $17,80+3,01$ & & $8,87+2,26$ & & $23,67+4,97$ & \\
\hline Estado civil & & 0,368 & & 0,673 & & 0,088 & & 0,474 \\
\hline Solteiro & $25,50 \pm 3,92$ & & $20,00 \pm 3,77$ & & $11,00+1,63$ & & $28,10 \pm 4,91$ & \\
\hline Casado & $24,09 \pm 3,61$ & & $19,82 \pm 4,46$ & & $9,82+2,22$ & & $27,01 \pm 6,16$ & \\
\hline Escolaridade & & 0,191 & & 0,379 & & 0,523 & & 0,223 \\
\hline Analfabeto & $24,66 \pm 4,12$ & & $20,03 \pm 4,79$ & & $9,97+2,63$ & & $28,14 \pm 6,75$ & \\
\hline Ensino fundamental & $23,44 \pm 2,87$ & & $19,69 \pm 4,29$ & & $9,63+1,71$ & & $25,25 \pm 5,08$ & \\
\hline Ensino médio & $23,36 \pm 3,57$ & & $19,03 \pm 3,98$ & & $9,69+1,97$ & & $26,39 \pm 5,37$ & \\
\hline Ensino superior & $26,67 \pm 3,06$ & & $22,67 \pm 2,08$ & & $11,33+0,058$ & & $28,00 \pm 3,46$ & \\
\hline Tempo cuidador & & 0,195 & & $0,017^{* *}$ & & $0,030^{* *}$ & & $0,013^{* *}$ \\
\hline$\leq 6$ & $24,64 \pm 4,30$ & & $19,91 \pm 3,53$ & & $10,36+2,16$ & & $27,73 \pm 4,94$ & \\
\hline 6а 12 & $24,62 \pm 3,74$ & & $19,50 \pm 4,05$ & & $9,85+1,90$ & & $25,27 \pm 5,72$ & \\
\hline 12 a 18 & $22,97 \pm 3,02$ & & $18,06 \pm 3,40$ & & $9,03+1,70$ & & $25,68 \pm 4,30$ & \\
\hline 18 a 24 & $24,93 \pm 4,67$ & & $22,80 \pm 5,76$ & & $10,93+3,11$ & & $31,60 \pm 8,02$ & \\
\hline$\geq 24$ & $23,25+2,22$ & & $21,75+2,99$ & & $11,25+1,50$ & & $28,50+3,42$ & \\
\hline
\end{tabular}

${ }^{*} p<0,05$ (** Teste de Kruskal-Wallis) (* Teste Mann Whitney-U). 


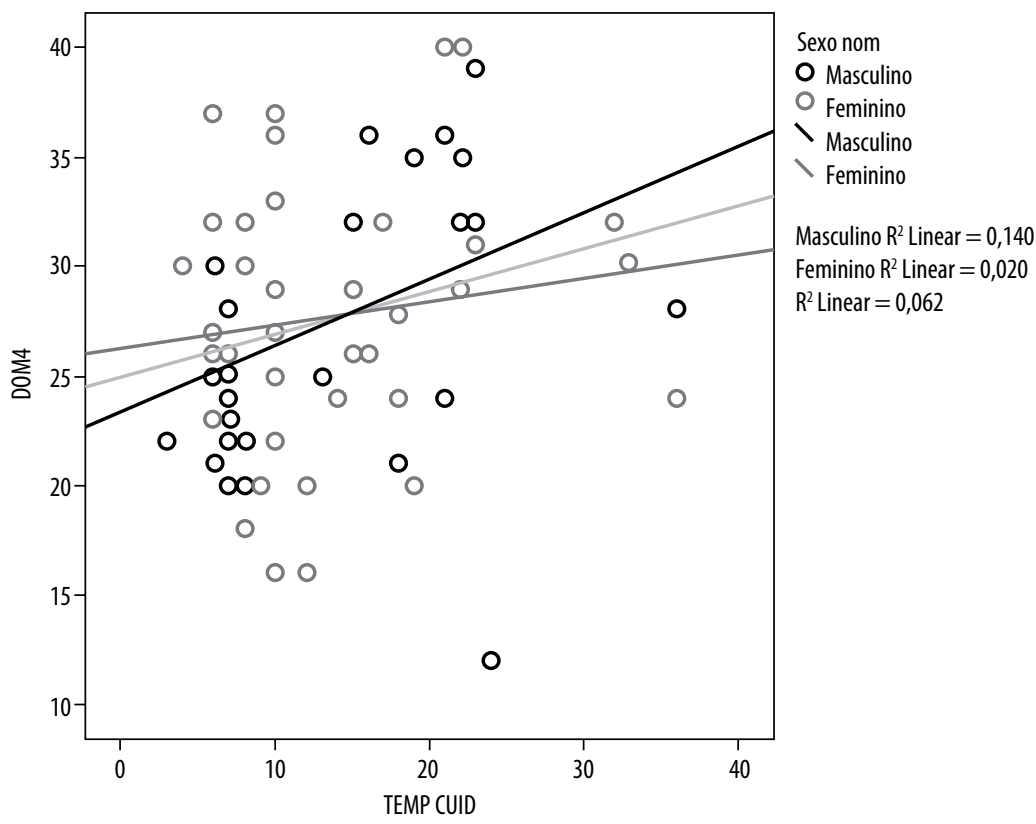

Figura 1. Análise de correlação entre o domínio Meio Ambiente (WHOQOL) e o tempo como cuidador, segundo o sexo Goiânia, GO, 2012 ( $n=90)$.

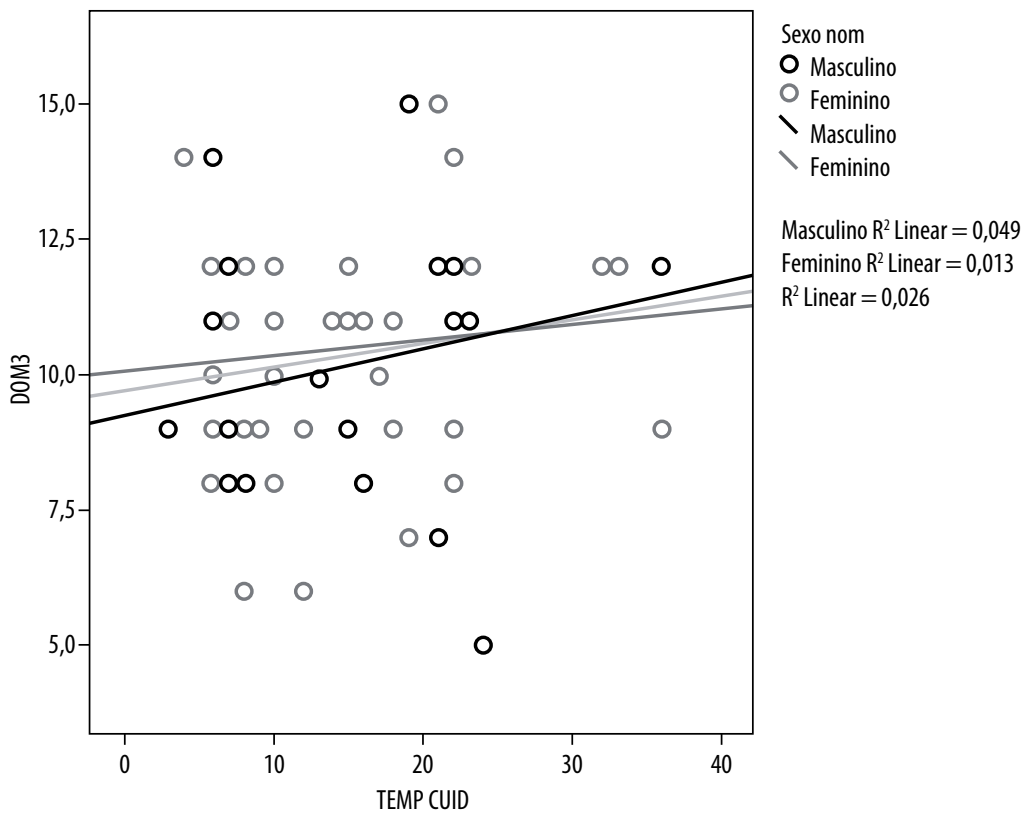

Figura 2. Análise de correlação entre o domínio Relações Sociais (WHOQOL) e o tempo como cuidador, segundo o sexo Goiânia, GO, 2012 ( $n=90)$

\section{DISCUSSÃO}

Estudos recentes têm demonstrado atenção com a qualidade de vida não só dos pacientes alvos das múltiplas terapêuticas, mas também daqueles que são os seus cuidadores. É possível identificar pesquisas envolvendo variadas patologias, entre elas aquelas classificadas dentro da especialidade da neurologia e psiquiatria. Apesar disso, não foi localizada nenhuma publicação especificamente relacionada com os cuidadores de pacientes com demência com corpos de Lewy.

Cuidar de um portador de alteração cognitiva tem sido culturalmente atividade atribuída ao sexo feminino ${ }^{13-15}$. À mulher é atribuído o papel social de organizadora do lar e ao homem, de manutenção financeira ${ }^{16}$. Neste estudo também houve distinção entre os sexos dos familiares cuidadores. 
O fato de a maioria ser casada era de se esperar, a exemplo de outros estudos com cuidadores ${ }^{13-15}$, considerando-se a idade média dos cuidadores do presente estudo. Chamou atenção o fato de haver um cuidador com 92 anos, o que também foi encontrado no estudo de Figueiredo et al., indicando que cada vez mais "idosos estão cuidando de idosos"119.

Difere, contudo, de outros estudos em que os cuidadores eram mais jovens que os portadores de demência ${ }^{18,16}$, mas ainda em torno dos 60 anos ${ }^{15}$.

A escolaridade dos cuidadores do presente estudo ficou entre ensino médio e analfabetos, corroborando outros estudos cuja média ficou entre $8^{14}$ e mais anos ${ }^{13}$ ou até quatro anos ${ }^{19}$ de estudos. A maior escolaridade foi associada ao maior escore da escala de avaliação subjetiva do impacto da demência de Alzheimer no cuidador ${ }^{16}$ e como fator que permite melhor compreensão da doença, em função do uso de recursos adequados para lidar com a situação e minimizar o impacto do cuidar ${ }^{20}$.

O baixo valor atribuído à média das respostas sobre a avaliação da qualidade de vida e satisfação com a saúde (overall) sugere que os cuidadores não estão satisfeitos, como também evidenciado no estudo de Figueiredo et al. ${ }^{19}$, que avaliou a satisfação com a vida e a percepção do estado de saúde em cuidadores de idosos com e sem demência. Esses autores consideram que cuidar durante tempo longo de um portador de demência pode ser física e psicologicamente esgotante e prejudicar a saúde e o bem-estar do cuidador. Há, inclusive, registros de que o cuidador do portador de Alzheimer busca os serviços médicos com maior frequência e utiliza mais psicotrópicos que os demais cuidadores ${ }^{21}$. A preocupação com a saúde do cuidador é pertinente, considerando-se que pode ser esse um dos fatores que contribui para a institucionalização do dementado.

A segunda maior média dos domínios do WHOQOL-BREF obtida no domínio Físico, que avalia as condições de locomoção, de energia física, de sono, de capacidade de trabaIho e de desempenho das atividades da vida diária, pode ser compreendida considerando-se a idade jovem dos cuidadores do estudo. Contudo, a baixa satisfação com a capacidade de desempenhar atividades cotidianas pode indicar que a preocupação constante e o tempo exigido para o cuidado ao portador de demência ${ }^{13,14}$ estão interferindo na vida do cuidador. Os estudos indicam que a sobrecarga é relacionada à presença de transtornos psiquiátricos dos portadores de demência 18,22 .

A menor média no domínio Físico foi dos cuidadores com idade acima de 60 anos, o que pode ser atribuído à fragilidade da idade, ao uso de medicações e ao tratamento médico ${ }^{23}$ e ao impacto do cuidado sobre a saúde do cuidador ${ }^{17}$. O trabalho de Pawlowski et al..$^{15}$ mostrou resultados semelhantes ao presente estudo, ao evidenciar que aqueles na faixa de idade entre 60 e 80 anos eram os que sofriam maior impacto ao realizar o cuidar de pacientes, com mais dores, desconforto, perda de energia, alteração do sono e sensação diminuída da capacidade de desempenhar atividades do dia a dia' ${ }^{15}$. Essa relação também foi evidenciada no trabalho de Valente et al. ${ }^{24}$, em que os cuidadores com problemas físicos foram mais velhos do que aqueles sem tais problemas.

Outros estudos têm apontado que os familiares cuidadores de idosos dementes relatam sentir mais desgaste, revolta, cansaço e depressão do que familiares de idosos em geral. As características próprias da doença que levam o doente a ter manifestações de agressividade e perda de domínio cognitivo produzem impacto sobre a qualidade de vida dos seus cuidadores. E quando tais cuidadores são os próprios familiares, isso parece ser ainda mais relevante, pois o ato de cuidar está envolvido com sentimentos e emoções. E muitas vezes tais sentimentos são conflitantes para o cuidador que se vê responsável por uma situação com a qual não sabe exatamente como lidar ${ }^{25}$.

Considerando-se que a sobrevida do portador de demência com corpos de Lewy é em torno de cinco $\operatorname{anos}^{26}$, é esperado que o tempo de cuidador não seja longo. A média do tempo obtida nos resultados desta pesquisa também foi encontrada em estudos que incluem portadores de síndrome demencial ${ }^{13}$, diferente daqueles realizados com cuidadores de portadores de demência de Alzheimer ${ }^{15}$, cuja sobrevida à doença é mais longa ${ }^{26}$.

Quando se relaciona o impacto na qualidade de vida dos cuidadores com o tempo como cuidador, observa-se que este pode ser um fator de perda na QV, influenciando nos domínios Psicológico, Meio Ambiente e Físico. Não há consenso nas pesquisas em relação ao impacto do tempo de cuidador ${ }^{21}$. Há estudos realizados com portadores de Alzheimer que indicam que com o passar do tempo o impacto tende a melhorar ${ }^{27}$, mas outros consideram que há uma piora ${ }^{13}$.

No presente estudo foi evidenciada piora das médias daqueles com menor tempo de cuidado, exceto no domínio Relação Social. Há de se considerar que esse resultado é limitado, por se tratar de uma coorte transversal, mas ele leva à hipótese de que o diagnóstico e a velocidade da evolução da demência com corpos de Lewy podem não ter permitido, ainda, adaptação dos cuidadores a essa nova condição. Alguns autores se referem às adaptações requeridas desde o ambiente físico à inversão de papéis no ambiente familiar, até as estratégias de gerenciamento de problemas do dementado, que interferem no ajuste emocional do cuidador, ou seja, a forma como ele interage com o dementado ${ }^{27,15}$, construindo um relacionamento pessoal diferenciado com ele.

A literatura indica a presença de depressão, sentimento de desprazer, alteração do interesse sexual, culpa e ideias suicidas $15,18,28,29$ nos cuidadores, principalmente associados à presença de transtornos psiquiátricos dos dementados. $\mathrm{O}$ fato de reconhecer que a doença é progressiva e que está 
diante de uma situação imutável, prolongada e que pode piorar também é apontado como fator que influencia na piora da qualidade de vida e saúde dos cuidadores ${ }^{29}$. Esses autores encontraram associação entre a qualidade de vida do cuidador e a boa saúde do dementado. No estudo de Lima-Costa et al. ${ }^{30}$, a variável insatisfação/muita insatisfação com os relacionamentos pessoais se relacionou positivamente com pior autoavaliação da saúde entre idosos, ressaltando a importância da boa interação entre cuidador e paciente. No nosso estudo o domínio Relação Social, que inclui aspectos sexuais, relações sociais e suporte social, foi o que apresentou menor média entre os domínios.

O isolamento social do cuidador também tem sido descrito como fator de impacto na qualidade de vida ${ }^{28}$, interferindo inclusive na sua saúde ${ }^{16}$.

Foram limitações do presente estudo a ausência de dados relativos às alterações comportamentais, que permitiriam melhor compreensão do impacto da tarefa de cuidar, a ausência de grupo controle e o fato de a natureza da amostra estudada ser de base ambulatorial, com escores provavelmente mais baixos nas escalas de sobrecarga em relação a uma amostra comunitária.

\section{CONCLUSÃO}

Os familiares cuidadores de portadores de demência com corpos de Lewy estudados são jovens, casados e com nível médio de escolaridade ou analfabetos.

A qualidade de vida desses cuidadores é afetada principalmente nos domínios Meio Ambiente, Físico e Psicológico. A melhor situação foi referida no domínio Meio Ambiente.

As variáveis associadas à qualidade de vida foram o sexo e o tempo de cuidador. As mulheres apresentaram pior escore no domínio Relação Social, mas apresentaram melhor escore no domínio Físico. E quanto mais tempo como cuidador, pior foi a qualidade de vida nos domínios Físico, Psicológico e Meio Ambiente.

É fundamental o suporte profissional aos aspectos emocionais e às alterações na saúde do cuidador, para que ele não se torne um "paciente oculto" e incapaz de lidar com as demandas do portador de demência com corpos de Lewy.

O conhecimento das especificidades e a compreensão dos sintomas dessa patologia também podem auxiliar na adequação dos recursos pessoais para o enfrentamento das alterações comportamentais, apontadas como o fator mais impactante na vida do cuidador.

Com base no envelhecimento humano, no número crescente de portadores de demências em geral e no consequente aumento do número de cuidadores, instrumentos específicos de avaliação poderiam ser desenvolvidos para possibilitar a definição das necessidades dos cuidadores e melhor planejamento de intervenções eficazes em reduzir os efeitos negativos no cotidiano deles.

\section{CONTRIBUIÇÕES INDIVIDUAIS}

Eberson Rodriques do Nascimento - Revisão teórica e sistematizada sobre o tema; elaboração e execução do projeto que originou o artigo; coleta dos dados, análise, discussão e revisão final do artigo.

Maria Alves Barbosa - Análise estatística dos dados, orientação na discussão dos resultados, revisão final do artigo.

Ana Luiza Lima Sousa - Análise estatística dos dados e orientação na discussão dos resultados.

Virginia Visconde Brasil - Análise e discussão.

Geraldo Francisco do Amaral - Análise e discussão.

Paula Jardem Jácomo - Coleta dos dados, análise e discussão.

\section{CONFLITOS DE INTERESSE}

Declaramos a não existência de conflitos de interesse e a não existência de fontes de financiamento para a realização deste trabalho.

\section{AGRADECIMENTOS}

Aos pacientes do Núcleo de Neurociência, sem os quais este trabalho não teria sido realizado, nosso profundo agradecimento pelo compromisso e pela participação.

E a todos que direta ou indiretamente contribuíram com este trabalho.

\section{REFERÊNCIAS}

1. Lopes MA, Bottino CMC. Prevalência de demência em diversas regiões do mundo: análise dos estudos epidemiológicos de 1994 a 2000. Arq Neuropsiquiatr. 2002;60(1):61-9.

2. Scazufca M, Cerqueira ATAR, Menezes PR, Prince M, Vallada HP, Miyazaki MCOS, et al. Investigações epidemiológicas sobre demência nos países em desenvolvimento. Rev Saúde Pública. 2002;36(6):773-8

3. Prince $M$, Acosta D, Chiu H, Scazufca M, Varghese M. Dementia diagnosis in developing countries: a cross-cultural validation study. Lancet. 2003;361(9361):888-9.

4. Papaléo Netto M. Tratado de gerontologia. 2ª ed. São Paulo: Atheneu; 2007.

5. Lewy FH. Paralysis agitans. I. Pathologische anatomie. Handbuch der Neurologie 3. Berlin: Springer; 1912. p. 920-33.

6. Kosaka K. Dementia and neuropathology in Lewy body disease. Adv Neurol. 1993;60:45663.

7. Byrne J. Doença de Parkinson e demência com corpúsculos de Lewy. In: Forlenza OV, Caramelli P. Neuropsiquiatria geriátrica. São Paulo: Atheneu; 2001. p. 215-20. 
8. Garre-Olmo J, López-Pousa S, Vilalta-Franch J, Turon-Estrada A, Hernández-Ferràndiz M, Lozano-Gallego M, et al. Carga do cuidador e sintomatologia depressiva em pacientes com a doença de Alzheimer: evolução aos doze meses. Rev Neurol. 2002;34:601-7.

9. Tavares A, Azeredo C. Demência com corpos de Lewy: uma revisão para o psiquiatra. Rev Psiq Clín. 2003;30(1):29-34.

10. The WHOQOL Group. The World Health Organization Quality of Life assessment (WHOQ0L): position paper from the World Health Organization. Soc Sci Med. 1995;41:1403-9.

11. McKeith IG, Dickson DW, Lowe J, Emre M, O'Brien JT, Feldman H, et al. Diagnosis and management of dementia with Lewy bodies: third report of the DLB Consortium. Neurology. 2005;65(12):1863-72.

12. Fleck MPA, Louzada S, Xavier M, Chachamovich E, Vieira G, Santos L, et al. Aplicação da versão em português do instrumento abreviado de avaliação de qualidade de vida WHOQ0L-BREF. Rev Saúde Pública. 2000;34(2):178-83.

13. Garrido R, Menezes PR. Impacto em cuidadores de idosos com demência atendidos em um serviço psicogeriátrico. Rev Saúde Pública. 2004;38(6):835-41.

14. Luzardo AR, Gorini MIPC, Silva APSS. Características de idosos com doença de Alzheimer e seus cuidadores: uma série de casos em um serviço de neurogeriatria. Texto \& Contexto Enferm. 2006;15:587-94

15. Pawlowski J, Gonçalvel TR, Hilgert JB, Hugo FN, Bozzetti MC, Bandeira DR. Depressão e relação com idade em cuidadores de familiares portadores de síndrome demencial. Estud Psicol. 2010;15(2):173-80

16. Lemos ND, Gazzola JM, Ramos LR. Cuidando do paciente com Alzheimer: 0 impacto da doença no cuidador. Saude Soc. 2006;15(3):170-9.

17. Karsch UM. Idosos dependentes: famílias e cuidadores. Cad Saude Publica. 2003;19(3):861-6.

18. Garrido R, Almeida OP. Distúrbios de comportamento em pacientes com demência: impacto sobre a vida do cuidador. Arq Neuropsiquiatr. 1999:57(2-B):427-34.
19. Figueiredo D, Lima MP, Sousa L. Os "pacientes esquecidos": satisfação com a vida e percepção de saúde em cuidadores familiares de idosos. Rev Kairós. 2009;12(1):97-112.

20. Conde-Sala JL, Garre-OImo J, Turró-Garriga 0, Vilalta-Franch J, López-Pousa S. Quality of life of patients with Alzheimer's disease: differential perceptions between spouse and adult child caregivers. Dement Geriatr Cogn Disord. 2010;29(2):97-108.

21. Cruz MN, Hamdan AC. 0 impacto da doença de Alzheimer no cuidador. Psicol Estud. 2008; $13(2): 223-9$

22. Levy K, Lanctôt KL, Farber SB, Li A, Herrmann N. Does pharmacological treatment of neuropsychiatric symptoms in Alzheimer's disease relieve caregiver burden? Drugs Aging. 2012;29(3):167-79.

23. Macedo C, Gazzola JM, Najas M. Síndrome da fragilidade do idoso. Arq Bras Ciênc Saúde. 2008:33(3):177-84

24. Valente LE, Truzzi A, Souza WF, Alves GS, Alves CE, Sudo FK, et al. Health self-perception by dementia family caregivers: sociodemographic and clinical factors. Arq Neuropsiquiatr. 2011;69(5):739-44.

25. Vitaliano PP, Zhang J, Scanlan JM. Is caregiving hazardous to one's physical health? A meta-analysis. Psychol Bull. 2003;129(6):946-72.

26. Ince PG, Perry EK, Morris CM. Dementia with Lewy bodies. A distinct non-Alzheimer dementia syndrome? Brain Pathol. 1998;8(2):299-324.

27. Hinrichsen $G A$, Niederehe $G$. Dementia management strategies and adjustment of family members of older patients. Gerontologist. 1994;34(1):95-102.

28. Vellone E, Piras G, Talucci C, Cohen MZ. Quality of life for caregivers of people with Alzheimer's disease. J Adv Nurs. 2008;61(2):222-31.

29. García-Alberca JM, Cruz B, Lara JP, Garrido V, Lara A, Gris E. Anxiety and depression are associated with coping strategies in caregivers of Alzheimer's disease patients: results from the MÁLAGA-AD study. Int Psychogeriatr. 2012;16:1-10.

30. Lima-Costa MF, Firmo JOA, Uchôa E. The structure of self-rated health among older adults: the Bambuí health and ageing study (BHAS). Rev Saúde Pública. 2004:38:827-34. 